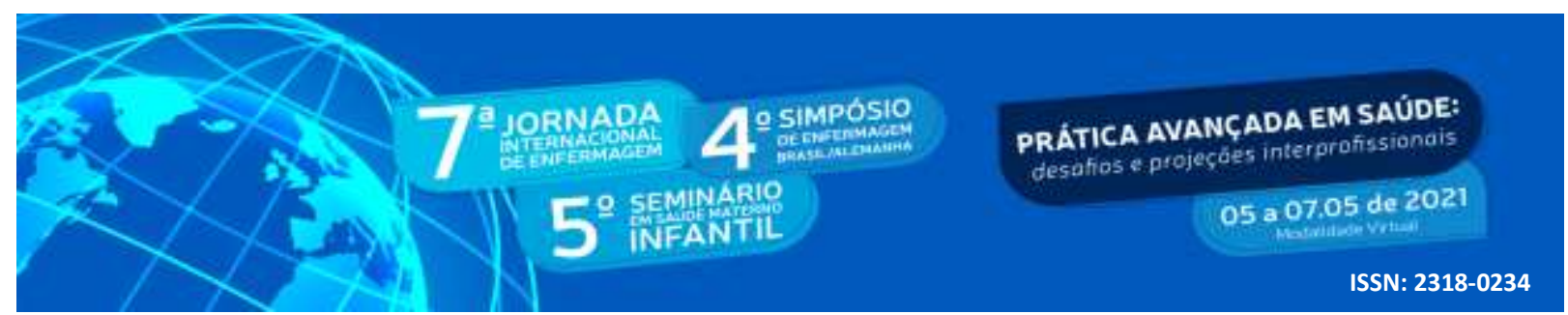

DOI: http://doi.org/10.48195/jie2021-017

\title{
USO DA TECNOSSOCIALIDADE NO QUOTIDIANO DE PROFISSIONAIS DA ATENÇÃO PRIMÁRIA EM TEMPOS DA PANDEMIA COVID-191
}

\author{
Tamires Carolina Silva²; Selma Maria da Fonseca Viegas ${ }^{3}$
}

\begin{abstract}
RESUMO
Este estudo teve como objetivo refletir sobre o uso da tecnossocialidade no quotidiano de profissionais da Atenção Primária à Saúde em tempos da pandemia de COVID-19. Trata-se de um ensaio teórico, uma reflexão sobre o uso das tecnologias digitais por profissionais de saúde, abrangendo as possibilidades do uso e os desafios enfrentados. Considera-se que as tecnologias virtuais têm potencial para ações em saúde e de interações no quotidiano para acompanhamento das pessoas, devem alçançar um espaço mais abrangente do que de um adicional serviço da rede de atenção à saúde após a pandemia. As ferramentas digitais como as mídias sociais, aplicativos móveis, telemedicina, teleconsultas e teleatendimento simbolizam fortes aliadas desses profissionais, demostrando-se eficácia para promover ações de promoção, proteção e educação em saúde, bem como, prevenção de agravos e riscos.
\end{abstract}

Palavras-chave: Atenção Primária à Saúde; Tecnologia; Rede Social; Profissionais de Saúde.

\begin{abstract}
This study aimed to reflect on the use of technosociality in the daily lives of Primary Health Care professionals in times of COVID-19 pandemic. This is a theoretical essay, a reflection on the use of digital technologies by health professionals, addressing the possibilities of use and the challenges faced. Considers that virtual technologies have the potential for health actions and interactions in the daily lives of people, should reach a more comprehensive space than an additional service in the health care network after a pandemic. Digital tools such as social media, mobile applications, telemedicine, teleconsultations and call centers symbolize strong allies of these professionals, demonstrating effectiveness in promoting health promotion, protection and education, as well as preventing injuries and risks.
\end{abstract}

Key Words: Primary Health Care; Technology; Social Media; Health Personnel.

\footnotetext{
${ }^{1}$ Ensaio Teórico. Financiamento: Coordenação de Aperfeiçoamento de Pessoal de Nível Superior (CAPES).

2 Enfermeira. Mestranda do Programa de Pós-Graduação em Enfermagem da Universidade Federal de São João del-Rei, Campus Centro-Oeste (UFSJ/CCO). E-mail: ta.csilva@ hotmail.com.

3 Enfermeira. Orientadora. Doutora e Pós-Doutora em Enfermagem. Docente Associado I da UFSJ/CCO, Divinópolis-MG, Brasil. E-mail: selmaviegas@ufsj.edu.br.
} 


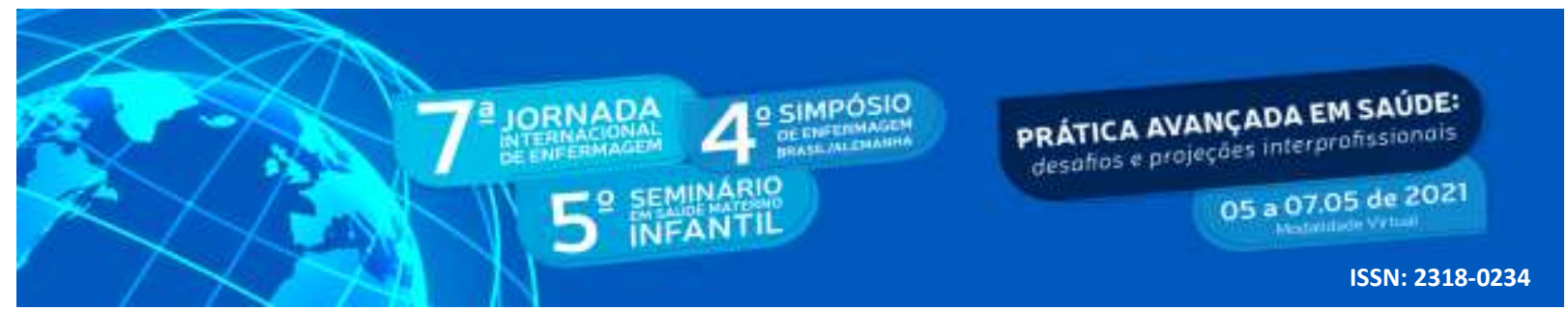

\section{INTRODUÇÃO}

Com as mudanças no quotidiano impactando na presença e na interação social pós-moderna, com o uso cada vez mais frequente de tecnologias para efetiva comunicação, a COVID-19 pausa o modo de viver e traz novas rotinas desde a notícia que o novo coronavírus (SARSCoV-2) teve sua origem na cidade de Wuhan na China, no final do ano de 2019. Com rápida disseminação o SARS-CoV-2 se espalhou rapidamente pelo mundo (BRUNS; HARRINGTON; HURCOMBE, 2020), sendo declarado o estado de pandemia de COVID-19 pela Organização Mundial da Saúde, em 12 de março de 2020 (WORLD HEALTH ORGANIZATION, 2020).

Com a pandemia o trabalho remoto e o fortalecimento da comunicação entre as pessoas, de forma virtual, se tornaram realidade, pois a infecção (COVID-19) enfrentada nos dias atuais, representa "um dos maiores desafios sanitários em escala global deste século" (WERNECK; CARVALHO, 2020, p. 01).

Visando conter a transmissão do SARS-CoV-2, medidas de saúde pública vêm sendo adotadas, dentre elas estão o isolamento social, quarentena e distanciamento social (LAKE, 2020). Tais medidas contribuem para o aumento do uso da internet, redes sociais virtuais e fórum online (OPAS, 2020). Ao mesmo tempo, que o desenvolvimento tecnológico contribui para o isolamento das pessoas, ele também tem contribuido para a constituição de "uma nova ligação: estar sempre, em contato, em união, em comunhão, ser antenado" (MAFFESOLI, 2012, p. 98)

Segundo Maffesoli a tecnossocialidade pode ser definida como uma forma de comunicação e interação social com uso da internet e de suas ferramentas (MAFFESOLI, 2016). Nota-se que "a pós-modernidade vem sendo construída na perspectiva de união, entrelace, estar-junto e de convivência entre o sagrado e profano, erudito e popular, razão e emoção, corpo e mente, ciência e arte, tradição e tecnologia" (CANDA, 2010, p. 70). Caracterizando assim, a vida pósmoderna como o ressurgir de um nova vida cotidiana e de novas maneiras de se estar junto por meio de tecnologia pós-moderna (MAFFESOLI, 2012; MAFFESOLI, 2010).

Na área da saúde, a utilização de ferramentas tecnológicas na Atenção Primária à saúde (APS), em tempos da pandemia de COVID-19, pode ser considerada uma poderosa aliada. Nota-se, que "as modalidades de atendimento online têm sido priorizadas e bastante difundidas" 


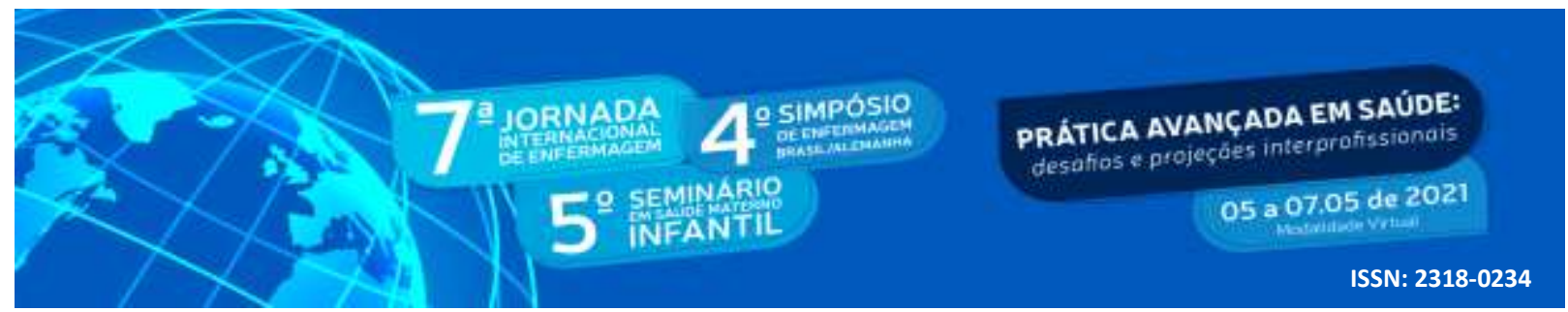

(MEDINA et al., 2020, p. 02). As tecnologias virtuais podem ser usadas pelos profissionais de saúde que atuam na APS durante este momento pandêmico vivenciado, para favorecer a realização de ações de promoção da saúde e prevenção de riscos e agravos (LIMA, 2020), educação em saúde (AUGUSTA; PALÁCIO; TAKENAMI, 2020), monitoramento de pacientes com COVID-19 em isolamento domiciliar (SANTOS; FRANÇA; SANTOS, 2020) e continuidade do cuidado ao paciente (MEDINA et al., 2020).

Contudo, a pandemia de COVID-19 tem causado grandes impactos e mudanças no quotidiano de trabalho de profissionais de saúde da APS (DAUMAS et al., 2020), dentre elas está a intensificação do processo de utilização (VITÓRIA; CAMPOS, 2020), conhecimento e aprendizagem de novas tecnologias digitais (DAVALBHAKTA et al., 2020), que merecem ser investigadas. A realização do presente ensaio justifica-se devido a necessidade de reflexão sobre o uso da tecnossocialidade no quotidiano de profissionais da APS em tempos da pandemia de COVID-19, bem como por ser uma temática atual mais ainda pouco explorada.

\section{OBJETIVO}

Refletir sobre o uso da tecnossocialidade no quotidiano de profissionais da APS, em tempos de COVID-19.

\section{METODOLOGIA}

O ensaio teórico possibilita a reflexão perante e análise do objeto/temática de estudo, independente de sua característica ou natureza (MENEGHETTI, 2011). Caracteriza-se por apresentar natureza interpretativa e reflexiva, diversificada da forma classificatória da ciência (MENEGHETTI, 2011; BERTERO, 2011). Nota-se, que "o ensaio precisa ser utilizado como opção consciente e intencional, ou seja, como a forma mais adequada no entendimento de algo" (MENEGHETTI, 2011, p. 323).

\section{DESENVOLVIMENTO (REFERENCIAL TEÓRICO)}

Os profissionais de saúde em linha de frente no enfrentamento da pandemia de COVID-19 


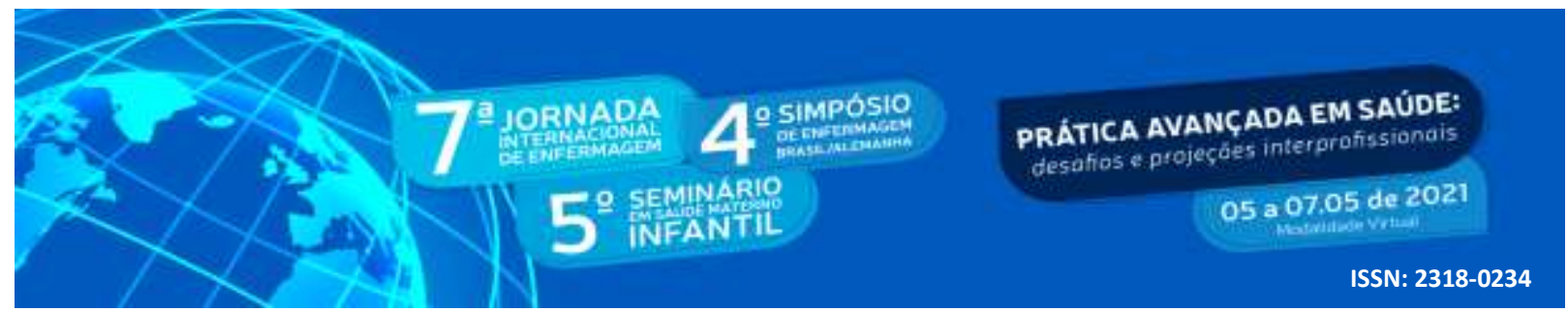

tiveram impacto sobre suas rotinas de trabalho e no seu viver, representam um grupo vulnerável relacionado ao alto risco de infecção (ALLEN et al., 2020; TEXEIRA et al., 2020). Profissionais da APS têm enfrentado quadros de ansiedade e excessivo medo, recorrentes ao momento vivenciado pela sobrecarga emocional e de trabalho (OLIVEIRA, 2020; CAETANO et al., 2020).

A APS é uma das portas de entrada do SUS, a prioritária, próxima das casas das pessoas e essencial na atenção à saúde frente a pandemia de COVID-19 (BARBOSA; SILVA, 2020). As equipes da APS desenvolvem ações de contenção da proliferação viral, guiando, sensibilizando e orientando as pessoas quanto à importância de adotarem as medidas protetivas (OLIVEIRA et al., 2021), bem como, prover a "sinalização da restrição de acesso da população às unidades de saúde para evitar a disseminação do novo Coronavírus" (OLIVEIRA, 2020, p. 13). Diante desse cenário, as tecnologias digitais surgem como grandes aliadas para aumentar o acesso, neste mundo moderno e cada vez mais digital (CARRER et al., 2020), Assim, instalando-se na sociedade pós-moderna uma nova maneira de se comunicar, por meio da internet (MAFFESOLI, 2010).

Nota-se, que a tecnologia pode ser usada para promover ações educativas e de comunicação em saúde. Segundo Maffesoli, a "comunicação e informação são etiquetas em voga. Ambas expressam conteúdos importantes da época atual" (MAFFESOLI, 2003, p. 14). Desta forma, os profissionais de saúde que atuam em equipes de saúde da família podem usar as mídias sociais como Facebook, Twitter e WhatsApp, para fornecer informações em saúde (CAETANO et al., 2020), orientando a população quanto às formas de contágio e sobre os disponíveis canais virtuais de teleatendimento (DAUMAS et al., 2020).

O WhatsApp representa uma ferramenta simples, que possibilita uma comunicação efetiva dos profissionais de saúde da APS. O fato de possibilitar o envio de mensagem de voz favorece o contato com indivíduos não alfabetizados, principalmente em comunidades de baixa renda (ALVES, 2020).

Observa-se, que os aplicativos móveis em saúde diante do impacto global da pandemia de COVID-19 persuadiu um desenvolvimento e utilização rápida. Esses aplicativos, de certa forma, estão abrangendo necessidades dos sistemas de saúde, como disseminação de informações de saúde, rastreamento de contatos, verificação de manifestações clínicas e 


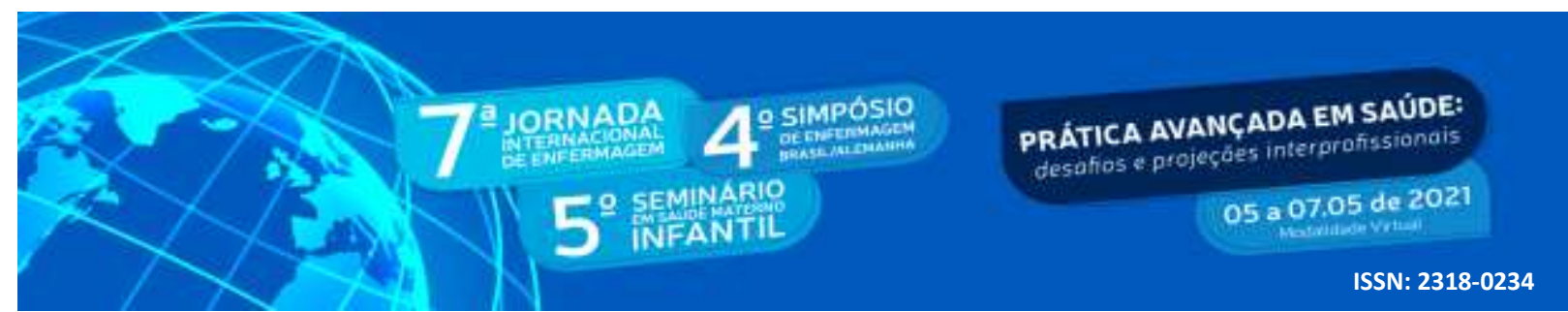

fornecimento de ferramentas para cursos e treinamentos de profissionais de saúde (DAVALBHAKTA et al., 2020).

Ressalta-se, que as tecnologias virtuais representam uma riqueza rara (MAFFESOLI, 2016), que não deveriam ser usadas somente neste período da pandemia de COVID-19 ou apenas como um adicional serviço da rede de atenção à saúde. A telemedicina oferece benefícios para o sistema, ressaltando a relevância de que estas sejam adotadas de maneira integral e abrangente na APS. Essa ferramenta digital pode produzir resultados positivos como acessibilidade, integralidade, resolubilidade e abrangência de cuidados, qualificação do monitoramento de pacientes em condições crônicas, quando implementada (SARTI et al., 2020). Além disso, a telemedicina reduz a exposição, o que protege tanto os médicos quanto pacientes (ANTHONY JNR., 2020).

Observa-se, que as consultas eletrônicas devem ser priorizadas neste momento da pandemia de COVID-19 (MAZZUCCHI et al., 2021). As tecnologias de comunicação e informação vêm sendo utilizadas para realização de consultas por meio da teleconsulta, já que as mesmas garantem a oferta de ações de maneira segura neste momento pandêmico. Além disso, é capaz de dar continuidade ao tratamento dos usuários, evitando assim o agravamento do quadro clínico por falta de acompanhamento. Pois, muitas vezes, é possível realizar avaliação clínica e responder a demandas por medicamentos de uso contínuo por vídeo consultas (DAUMAS et al., 2020; MEDINA et al., 2020).

Os profissionais de saúde da APS podem usar a modalidade de teleatendimento para acompanhar e orientar casos suspeitos e confirmados de COVID-19, quando necessário o isolamento social dos pacientes e (re)conhecimento de sinais de alerta. Essa ferramenta também pode ser usada para identificar os clientes que podem ser cuidados em ambiente domiciliar, e em casos de sinais de agravamento solicitar a remoção para uma unidade hospitalar (DAUMAS et al., 2020; SANTOS; FRANÇA; SANTOS, 2020; CAETANO et al., 2020).

Por meio do uso da telemedicina, os profissionais de saúde que atuam na APS podem fornecer cuidados domiciliares a pacientes com câncer avançado durante a pandemia. Essa tecnologia audiovisual permite que os profissionais façam videochamadas, chamadas de voz e mensagens de texto, tornando o processo mais conveniente e dinâmico. A maior dificuldade enfrentada pelos profissionais de saúde foi fornecer acompanhamento contínuo de qualidade 


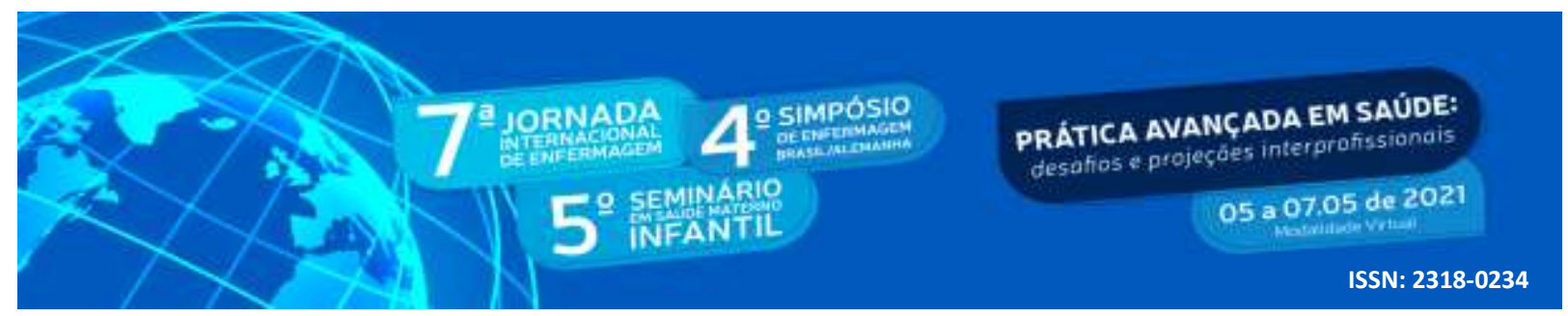

aos pacientes em estado paliativo (BISWAS; ADHIKARI; BHATNAGAR, 2020).

Dentre os desafios enfrentados pelos profissionais de saúde da APS no uso de tecnologias digitais estão a necessidade de uma internet de qualidade (ALVES, 2020), falta de confiança de alguns profissionais, falta de prontuário eletrônico que interaja com o sistema e-SUS (SARTI et al., 2020).

Contudo, no contexto de pandemia para que essas mudanças necessárias sejam implementadas ao pleno funcionamento da APS, são necessários investimentos em estruturas, como telefones, internet de boa qualidade (DAUMAS et al., 2020; CAETANO et al., 2020; SARTI et al., 2020) e computadores (SARTI et al., 2020), capazes de assegurar o serviço, sistema, recursos humanos, modelo de organização e a interoperabilidade. Sua implementação também demanda investimento de tempo para a sua constituição (CAETANO et al., 2020)

\section{CONCLUSÃO}

Em suma considera-se que, com a inesperada pandemia de COVID-19 tornou-se ainda mais evidente, o que já havia sendo discutido na literatura sobre o potencial do uso das tecnologias digitais em saúde. Desta forma, as ferramentas digitais representam fortes aliadas que vêm cada vez mais conquistando espaço no quotidiano de profissionais de saúde e dos serviços de saúde, demonstrando a eficácia nas ações de proteção, educação e promoção da saúde, como na prevenção de riscos e agravos.

\section{REFERÊNCIAS}

ALlEN, W. E.; ALTAE-TRAN, H.; BRIGGS, J.; JIN, X.; MCGEE, G.; SHI, A. et al.Population-scale longitudinal mapping of COVID-19 symptoms, behaviour and testing. Nature Human Behavion, v. 04, p. 972-982, sept. 2020. https://doi.org/10.1038/s41562-02000944-2

ALVES, M. T. G. Reflexões sobre o papel da Atenção Primária à Saúde na pandemia de COVID-19. Revista Brasileira de Medicina de Família e Comunidade, v. 15, n. 42, 05 p., jul. 2020. https://doi.org/10.5712/rbmfc15(42)2496

ANTHONY JNR., J. Use of Telemedicine and Virtual Care for Remote Treatment in Response to COVID-19 Pandemic. Journal of Medical Systems, v. 44, n. 07, 44-132 p., 2020 Jun. https://doi.org/10.1007/s10916-020-01596-5 


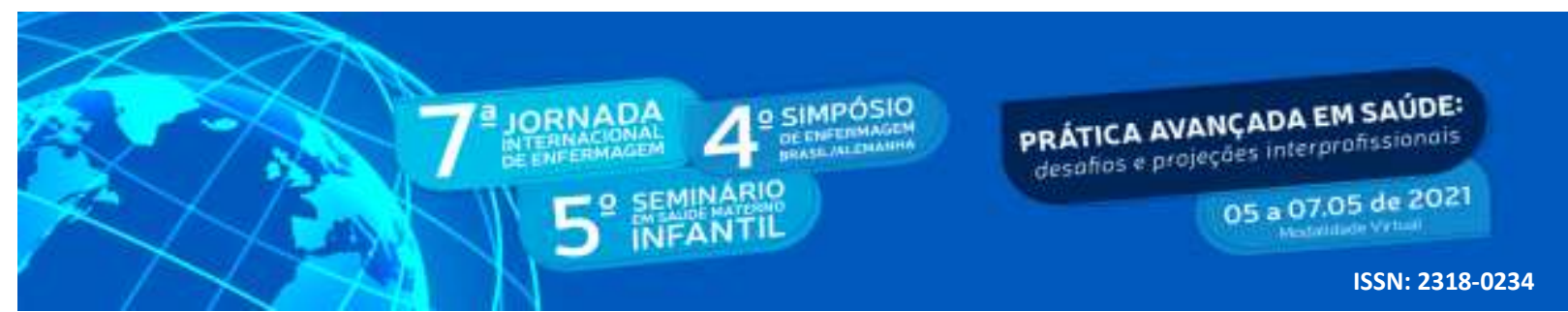

AUGUSTA, M.; PALÁCIO, V.; TAKENAMI, L. In times of pandemic by COVID-19: the challenge for health education. Vigilância Sanitária em Debate, v. 8, n. 2, p. 10-15, 2020 Abr. https://doi.org/10.22239/2317-269x.01530

BARBOSA, S. DE P.; SILVA, A. V. F. G. A Prática da Atenção Primária à Saúde no Combate da Covid-19. APS em Revista, v. 2, n. 1, p. 17-19, jan./abr. 2020. Disponível em: https://profsaude-

abrasco.fiocruz.br/sites/default/files/publicacoes/a_pratica_da_atencao_primaria_a_saude_no _combate_da_covid-19.pdf

BERTERO, C. O. Réplica 2 - O Que é um Ensaio Teórico? Réplica a Francis Kanashiro Meneghetti. RAC - Revista de Administração Contemporânea, v. 15, n. 2, mar./abr. 2011, pp. 338-342. Disponível em: https://www.redalyc.org/pdf/840/84018474012.pdf

BISWAS, S.; ADHIKARI, S.; BHATNAGAR, S. Integration of telemedicine for home-based end-of-life care in advanced cancer patients during nationwide lockdown: A case series. Indian Journal of Palliative Care, v. 26, n. 5, 176-178 p., 2020 Jun. https://doi.org/10.4103/IJPC.IJPC_174_20

BRUNS, A.; HARRINGTON, S.; HURCOMBE, E. 'Corona? 5G? or both?': the dynamics of COVID-19/5G conspiracy theories on Facebook. Media International Australia, v. 177, n. 01, 12-29 p., 2020. https://doi.org/10.1177\%2F1329878X20946113

CAETANO, R.; SILVA, A. B.; GUEDES, A. C. C. M.; PAIVA, C. C. N. DE; RIBEIRO, G. DE R.;SANTOS, D. L. et al. Desafios e oportunidades para telessaúde em tempos da pandemia pela COVID-19: uma reflexão sobre os espaços e iniciativas no contexto brasileiro. Caderno de Saúde Pública, v. 36, n. 05, 2020. https://doi.org/10.1590/0102-311X00088920

CANDA, C. N. Lá vai a vida a rodar: reflexões sobre práticas cotidianas em Michel Maffesoli. Rascunhos Culturais, v. 01, n. 02, p. 63-77, 2010. Disponível em: http://revistarascunhos.sites.ufms.br/files/2012/07/2ed_artigo_4.pdf

CARRER, F. C. DE A.; MATUCK, B. F.; LUCENA, E. H. G. DE; MARTINS, F. C.; JUNIOR, G. A. P.; GALANTE, M. L. et al. Teleodontologia e SUS: uma importante ferramenta para a retomada da Atenção Primária à Saúde no contexto da pandemia de COVID-19. Pesquisa Brasileira em Odontopediatria e Clínica Integrada, v. s., n. s., jun. 2020. https://doi.org/10.1590/SciELOPreprints.837

DAUMAS, R. P.; SILVA, G. A. E; TASCA, R.; LEITE, I. DA C.; BRASIL, P.; GRECO, D. B. et al. The role of primary care in the Brazilian healthcare system: limits and possibilities for fighting COVID-19. Cad. Saúde Pública, v. 36, n. 06, 2020 Jun. http://dx.doi.org/10.1590/0102-311X00104120

DAVALBHAKTA, S.; ADVANI, S.; KUMAR, S.; AGARWAL, V.; BHOYAR, S.; FEDIRKO, E. et al. A Systematic Review of Smartphone Applications Available for Corona Virus Disease 


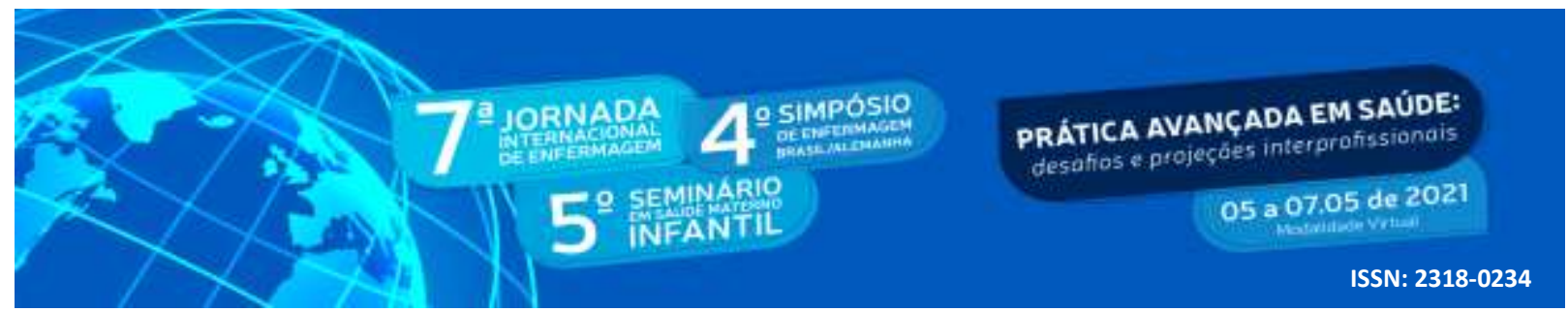

2019 (COVID19) and the Assessment of their Quality Using the Mobile Application Rating Scale (MARS). Journal of Medical Systems volume, v. 44, n. 09, 44-164 p., 2020 Aug. https://doi.org/10.1007/s10916-020-01633-3

LAKE, M. A. What we know so far: COVID-19 current clinical knowledge and research. Clinical Medicine, v. 20, n. 2, p. 124-127, 2020 Mar. https://dx.doi.org/10.7861\%2Fclinmed.2019-coron

LIMA, R. C. Distanciamento e isolamento sociais pela Covid-19 no Brasil: impactos na saúde mental. Physis, Rio de Janeiro, v.30 n.2, 2020 Abr./Jul. http://dx.doi.org/10.1590/S010373312020300214

MAFFESOLI, M. A comunicação sem fim (teoria pós-moderna da comunicação). Revista FAMECOS, Porto Alegre, v. 10, n. 20, abril 2003. https://doi.org/10.15448/19803729.2003.20.3198

MAFFESOLI, M. A ordem das coisas: pensar a pós-modernidade. Rio de Janeiro: Forense, 2016.

MAFFESOLI, M. O tempo retorna: formas elementares da pós-modernidade. Rio de Janeiro: Forense Universitária, 2012.

MAFFESOLI, M. Saturação. São Paulo: Iluminuras: Itaú Cultura, 2010. Disponível em: http://d3nv1jy4u7zmsc.cloudfront.net/wp-content/uploads/itau_pdf/001733.pdf

MAZZUCCHI, E.; TORRICELLI, F. C. M.; VICENTINI, F.; MARCHINI, G.; DANILOVIC, A.; BATAGELLO, C. et al. The impact of COVID-19 in medical practice. A review focused on Urology. Int. braz j urol., Rio de Janeiro, v. 47, n.2, 251-262 p., 2021 Mar. http://dx.doi.org/10.1590/s1677-5538.ibju.2020.99.08

MEDINA, M. G.; GIOVANELlA, L.; BOUSQUAT, A.; MENDONÇA, M. H. M. DE; AQUINO, R. Atenção primária à saúde em tempos de COVID-19: o que fazer? Cad. Saúde Pública, v.36, n. 8, 2020. https://dx.doi.org/10.1590/0102-311X00149720

MENEGHETTI, F. K. et al. O que é um Ensaio-Teórico? RAC, Curitiba, v. 15, n. 2, p. 320332, mar./abr. 2011. https://doi.org/10.1590/S1415-65552011000200010

OLIVEIRA, L. M. S. DE; GOMES, N. P.; OLIVEIRA, E. S.; SANTOS, A. DE A.; PEDREIRA, L. C. Coping strategy for covid-19 in primary health care: experiencereport in Salvador-BA. Rev Gaúcha Enferm., v. 42, n. 01, 2021 Jan. https://doi.org/10.1590/19831447.2021.20200138

OLIVEIRA, G. S. DE; MONTERIRO, L. De S.; CARVALHO, M. DE A. A.; FREIRE, A. K. DA S. Mental Health in the progress of the COVID-19 Pandemic: Conceptions of Primary Health Care workers. Research, society and development, v. 09, n. 10, 2020. https://doi.org/10.33448/rsd-v9i10.9339 


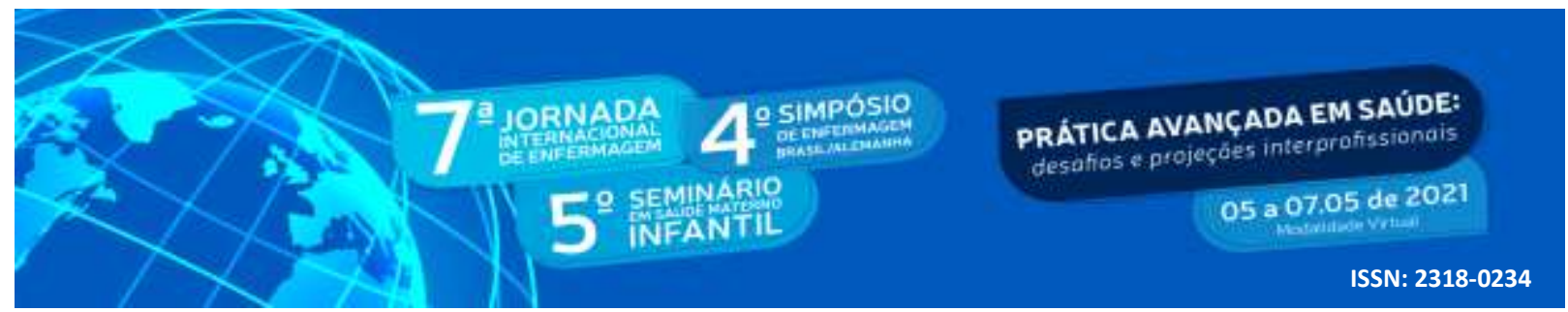

OPAS. Entenda a infodemia e a desinformação na luta contra a COVID-19. C. s.: Organização Mundial de Saúde, 2020. Disponível em: https://iris.paho.org/bitstream/handle/10665.2/52054/Factsheet-

Infodemic_por.pdf?sequence $=14 \&$ isAllowed $=y$

SANTOS, A. B. S. DOS; FRANÇA, M. V. S.; SANTOS, J. L. F. DOS. Atendimento remoto na APS no contexto da COVID-19: a experiência do Ambulatório da Comunidade da Escola Bahiana de Medicina e Saúde Pública em Salvador, Bahia. APS em Revista, v. 2, n. 2, p. 169176, jun. 2020. https://doi.org/10.14295/aps.v2i2.120

SARTI, T. D.; LAZARINI, W. S.;FONTENELLE, L. F.; ALMEIDA, A. P. S. C. Qual o papel da Atenção Primária à Saúde diante da pandemia provocada pela COVID-19? Epidemiol. Serv. Saude, Brasília, v. 29, n. 2, p. 05, 2020. https://doi.org/10.5123/S1679-49742020000200024

TEIXEIRA, C. F. DE S.; SOARES, C. M.; SOUZA, E. A.; LISBOA, E. S.; PINTO, I. C. DE M.; ANDRADE, L. R. DE et al. The health of healthcare professionals coping with the Covid19 pandemic. Ciência e Saúde Coletiva, Rio de Janeiro, v. 25, n. 09, 2020 Aug./Sept. https://doi.org/10.1590/1413-81232020259.19562020

VITÓRIA, A. M.; CAMPOS, G. W. DE S. APS forte para achatar a curva da pandemia e garantir suficiência de leitos UTI. Conselho Nacional de Secretarias Municipais de Saúde, v. s., n. $\quad$ s., 8 p., 2020. Disponível em: http://www.cosemssp.org.br/wpcontent/uploads/2020/04/So-APS-forte-para-ter-leitos-UTI-.pdf

WERNECK, G. L.; CARVALHO, M. S. A pandemia de COVID-19 no Brasil: crônica de uma crise sanitária anunciada. Caderno de Saúde Pública, v. 36, n. 05, 2020. https://doi.org/10.1590/0102-311X00068820

WORLD HEALTH ORGANIZATION. WHO announces COVID-19 outbreak a pandemic. Denmark: Wordl Health Organization, marc. 2020. Available in: http://www.euro.who.int/en/health-topics/healthemergencies/coronavirus-covid-19/news/ 